\title{
El que con niñas se acuesta... denunciado amanece. Caracterización de las relaciones impropias en Costa Rica durante el periodo 2000-2018
}

\author{
Ángel Jesús Porras-Solis* \\ (iD) https://orcid.org/0000-0002-9394-1912 \\ Maritza Rodríguez-Soto** \\ iD https://orcid.org/0000-0002-7083-5260
}

\author{
Laura Solís-Bastos*** \\ (iD) https://orcid.org/0000-0002-7434-221X \\ Kimberley Contreras-Castro**** \\ (iD https://orcid.org/0000-0003-2060-5931
}

Porras-Solís, Á., Rodríguez-Soto, M., Solís-Bastos, L. y Contreras-Castro, K. (2020). El que con niñas se acuesta... denunciado amanece. Caracterización de las relaciones impropias en Costa Rica durante el periodo 2000-2018. Revista Espiga, 19 (39), 77-98.

\section{Formato de citación según Chicago-Deusto}

Porras-Solís Ángel Jesús, Maritza Rodríguez-Soto, Laura Solís-Bastos y Kimberley Contreras-Castro (2020). «El que con niñas se acuesta... denunciado amanece. Caracterización de las relaciones impropias en Costa Rica durante el periodo 2000-2018». Revista Espiga 19, n. ${ }^{\circ} 39$ (enero-junio, 2020): 77-98.

* Licenciado en Economía por la Universidad Nacional (UNA), de Costa Rica. Máster en Técnicas Estadísticas por la Universidad de Santiago Compostela, España. Se desempeña como docente e investigador en el Instituto de Estudios Sociales en Población (IDESPO-UNA). Correo: angel.porras.solis@ una.ac.cr

** Licenciada en Psicología por la Universidad de Costa Rica (UCR). Máster en Psicología Clínica Grupal. Investigadora del Programa Umbral Político, del Instituto de Estudios Sociales en Población (IDESPOUNA), de Costa Rica. Correo: maritza.rodriguez.soto@una.ac.cr

*** Licenciada en Sociología por la Universidad Nacional (UNA), de Costa Rica. Máster en Estudios Latinoamericanos con énfasis en cultura y desarrollo. Investigadora del Programa Umbral Político, del Instituto de Estudios Sociales en Población (IDESPO-UNA), de Costa Rica. Correo: laura.solis.bastos@ una.ac.cr

**** Bachiller en Relaciones Internacionales por la Universidad Nacional (UNA), de Costa Rica. Estudiante asistente del Programa Umbral Político, del Instituto de Estudios Sociales en Población (IDESPO-UNA), de Costa Rica. Correo: kimberly.contrerascastro@gmail.com 


\section{RESUMEN}

En Costa Rica fue aprobada la Ley 9406 en noviembre de 2016, conocida como «Ley de relaciones impropias»; estas son definidas por un vínculo de pareja marcado por las diferencias amplias de edades entre sus miembros. Mediante el análisis de los datos disponibles para el periodo 20002018, se llevó a cabo una revisión de fuentes de información, como las estadísticas de nacimientos y matrimonios, obtenidas en el sistema de consulta de indicadores del Instituto Nacional de Estadística y Censos (INEC), mediante REDATAM 7.

Este artículo aborda este fenómeno desde dos aristas: las uniones mediante el matrimonio y los embarazos adolescentes; en ambos casos, al filtrar la información disponible a partir de la identificación de una relación impropia, permitió caracterizar el comportamiento durante el periodo de estudio, lo que muestra que, una vez aplicadas medidas políticas, es posible incidir en las estadísticas de población. Sin embargo, se debe destacar que las estadísticas podrían tener limitaciones en tanto la cobertura de recolección de datos sobre este fenómeno podría estar siendo ocultada, u omitida, como una medida de protección por las adolescentes al momento de su declaración.

Palabras clave: Relaciones impropias, embarazo adolescente, abuso de poder, estadísticas de población adolescente, ley contra las relaciones impropias.

\section{Introducción}

Este artículo procura abordar el fenómeno de las relaciones impropias en Costa Rica; primeramente, sobre las implicaciones de las relaciones impropias como manifestaciones de abuso de poder; posteriormente, se hace una descripción acerca de la «Ley de relaciones impropias», aprobada en Costa Rica; y, finalmente, se incluye un análisis de las estadísticas de nacimientos y matrimonios en el país, en el marco de relaciones impropias durante el periodo 2000-2018, así como las estadísticas de denuncias por delitos vinculados a la Ley 9406, publicadas por el Poder Judicial.

En el marco de este artículo, es necesario mencionar el contexto actual del tema, en tanto las relaciones impropias, el cual se aclara a partir de la aprobación de la Ley 9406 para el fortalecimiento de la protección legal de las niñas y las adolescentes mujeres ante situaciones de violencia de género, asociadas a relaciones abusivas; ley aprobada por la Asamblea Legislativa de la República de Costa Rica y que entró en vigencia en enero de 2017, con la cual se ratifica que este tipo de relaciones debe ser considerado como una forma de violencia contra las personas menores de edad, principalmente para el caso de niñas y adolescentes.

Este fenómeno provoca implicaciones para las niñas y adolescentes que viven esta experiencia, en el tanto estas relaciones conllevan, en la mayoría de los casos, manifestaciones de dinámicas de poder desiguales, en las cuales la diferencia etaria pesa entre los factores vinculados al poder patriarcal, lo cual favorece a la persona de mayor edad, particularmente a los hombres, tal como se señala en este estudio.

En el marco de esta coyuntura específica, se torna relevante llevar a cabo el análisis de las estadísticas de nacimientos y las estadísticas de inscripción de matrimonios para el periodo 2000-2018, con el foco principal en los años 2017 y 2018, en los cuales se reflejan datos que podrían asociarse a los primeros efectos de la Ley 9406, en tanto se logra 
identificar las variaciones en la tendencia de los casos cuyas características determinan las relaciones impropias según lo estipulado por la ley.

\section{Metodología}

Como fuente principal de información se utiliza el registro de estadísticas vitales del Instituto Nacional de Censos (INEC) de Costa Rica, el cual designa todos los hechos relacionados con nacimientos, matrimonios y defunciones en el país. Para el presente trabajo, solo se toman como referencia las estadísticas de nacimientos y matrimonios.

En el caso particular de las estadísticas de nacimientos, estas permiten analizar la frecuencia con que estos se dan en todo el país, así como la obtención de información en otros niveles de agregación, tales como edad, nacionalidad, estado conyugal y ocupación de la madre y del padre.

Por su parte, las estadísticas de matrimonio surgen del procesamiento de la información obtenida sistemáticamente a partir de los certificados de matrimonio, lo cual permite realizar tabulaciones y análisis por grupos de edades, tipo de unión, condición de actividad y ocupación de la contrayente y del contrayente, estado conyugal de la contrayente y del contrayente, lugar de ocurrencia de la unión (provincia, cantón y distrito).

En ambos casos se analizaron los datos referentes a los rangos de edad de las madres y padres (nacimientos); así como de las contrayentes y de los contrayentes, hecho que dan cuenta de una relación impropia, mediante el programa de procesamiento de datos REDATAM 7 del INEC.

Como información complementaria para el análisis, se consultó las estadísticas públicas disponibles en el Observatorio de violencia de género contra las mujeres y acceso a la justicia del Poder Judicial, esto para identificar las denuncias interpuestos en el Ministerio Público por delitos como: abuso sexual contra personas menores de edad e incapaces; y relaciones sexuales con personas menores de edad, durante el periodo de estudio.

\section{Desarrollo. Implicaciones de las relaciones impropias, manifestaciones de abuso de poder}

Las relaciones impropias, según el Fondo de Población de Naciones Unidas (UNFPA, por su siglas en inglés $)^{1}$, se conciben como «(...)“uniones impropias”, el cual devela relaciones de poder, en estos casos, entre un hombre adulto y una niña o adolescente menor de edad, y los vínculos entre esas relaciones con el embarazo de niñas y adolescentes en Costa Rica».

$\mathrm{Al}$ abordar la problemática de las relaciones impropias, surge la pregunta respecto a las personas mayores de edad con las que jóvenes menores de edad establecen relaciones

1. Fondo de Población de Naciones Unidas, Uniones Tempranas y Embarazo en la Niñez y la Adolescencia en Costa Rica (2014): 13, acceso: 26 de marzo, 2020, http://tiempodehablar.paniamordigital.org/files/ Descargas/files/4_221_unionesimpropiasunfpapaniamor2014.pdf 
de convivencia. Según el UNFPA², existen pocos estudios que brindan respuesta a esta interrogante; no obstante, los datos del Registro de Matrimonios de Costa Rica (2011) y el X Censo Nacional de Población y VI de Vivienda (2011), del INEC, refieren que en las parejas con relación de parentesco, jefe-conyugue del hogar, alrededor del $98 \%$ de las adolescentes convive con hombres mayores de edad. La edad de los hombres que contraen matrimonio con adolescentes, en su mayoría, está «particularmente, entre los 20 y 24 años. Aproximadamente, un tercio de los hombres con este comportamiento son mayores de 25 años, y destaca el porcentaje de hombres mayores de 30 años de edad $(10.2 \%) »^{3}$.

Sin pretender establecer generalizaciones, surge la interrogante respecto de cuáles son los posibles aspectos, condicionantes, que podrían determinar un vínculo de este tipo. Para efectos de este trabajo, se rescata desde distintas posturas teóricas que abordan la temática, lo referente al sistema patriarcal y las relaciones de poder.

En lo que respecta al sistema patriarcal, autores como Fernández y Duarte ${ }^{4}$ señalan que el patriarcado es un orden social que se fundamenta en relaciones asimétricas de poder, donde lo masculino se antepone a lo femenino como superior y dominante, lo que se sustenta y legitima en preceptos sociales y en las instituciones que los representan. De igual forma, Castells, citado por Montesó ${ }^{5}$, afirma que:

el patriarcado es una de las estructuras básicas de todas las sociedades contemporáneas. Se caracteriza por la autoridad, impuesta desde las instituciones, de los hombres sobre las mujeres y sus hijos, en la unidad familiar. Para Castells es el patriarcado el principal elemento en la dominación de la mujer.

Según León ${ }^{6}$, al citar a la Organización Mundial de la Salud (OMS), la violencia contra las mujeres «tiene consecuencias negativas sobre la salud, que abarca la salud física, mental, sexual y reproductiva, e implica grandes aumentos en los niveles de morbilidad y mortalidad de las mujeres».

De acuerdo con Villareal ${ }^{7}$, al citar a Lagarde, las relaciones de poder del sistema patriarcal se presentan en todos los niveles de la sociedad, tanto en el ámbito privado como

2. Ibíd., 13 .

3. Ibíd., 14.

4. Fernández Carballo, Rodolfo y Andrea Duarte Cordero, «Preceptos de la ideología patriarcal asignados al género femenino y masculino, y su refractación en ocho cuentos utilizados en el Tercer Ciclo de la Educación General Básica del Sistema Educativo Costarricense en el año 2005», Revista Educación 30 (2) (2006), acceso: 26 de marzo, 2020, https://revistas.ucr.ac.cr/index.php/educacion/article/view/2235

5. Montesó Curto, Pilar, «Dificultades para el avance de las mujeres. Diferentes teorías sociológicas», Enfermería Global, Vol. 13, Núm. 4 (2014): 266, acceso: 26 de marzo, 2020, http://revistas.um.es/eglobal/ article/view/186761

6. León Camacho, Jennyfer Akira, «Propuesta educativa en materia de violencia contra las mujeres en relaciones de pareja, dirigida a centros educativos, comunidades y oficiales de la Fuerza Pública: un abordaje por medio de la oficina de la mujer del cantón central de Limón» (tesis de licenciatura en Psicología, Universidad de Costa Rica, 2018): 4, acceso: 26 de marzo, 2020, http://repositorio.sibdi.ucr.ac.cr:8080/ xmlui/handle/123456789/6550

7. Villarreal Montoya, Ana Lucía, «Relaciones de poder en la sociedad patriarcal», Revista Electrónica Actualidades Investigativas en Educación, vol. 1, no. 1 (2001): 3, acceso: 26 de marzo, 2020, https://revistas. ucr.ac.cr/index.php/aie/article/view/8456 
público. Estos poderes de dominio son «(...) sociales, grupales y personales, permiten explotar y oprimir a personas y grupos y todo tipo de colectividades. Se concretan en procesos concatenados de formas de intervenir en la vida de otras/os desde un rango y una posición de superioridad (valor, jerarquía, poderío)».

Según Ulate, citada por León ${ }^{8}$, el maltrato que sufren las mujeres en muchas ocasiones está favorecido por la falta de independencia, ya que a pesar de contar con recursos económicos, una mayoría de mujeres no logra alcanzar autonomía personal debido a la «construcción social». En este sentido, León ${ }^{9}$ señala:

Los seres humanos, a pesar de ser individuos, están inmersos en una sociedad que los influye en la forma de actuar, pensar y relacionarse. Lamentablemente, dicha sociedad fomenta las relaciones desiguales entre hombres y mujeres, en las cuales existe una dinámica de poder que naturaliza y justifica la violencia contra las mujeres.

No obstante, aunque el patriarcado establece una relación en la cual el hombre subordina a la mujer, resulta importante rescatar que, de acuerdo con lo estipulado por Villareal ${ }^{10}$, en las relaciones de poder, quienes participan, no carecen totalmente de este, a pesar de que existen pocos estudios que desglosen con cuales cuentan las mujeres; por el contrario, se profundiza en los roles como subordinadas y víctimas, lo que imposibilita identificar sus poderes, aunque limitados, para repensar dichas relaciones.

Por lo anterior, según Villareal1", «el término "relaciones de poder" implica de hecho que se da entre dos o más personas, quienes establecen dicha conexión porque necesitan satisfacer alguna necesidad. Esto implica a su vez que ambas personas aportan algo, unos poseen lo que las otras no tienen.». Por tanto ${ }^{12}$, «en la sociedad patriarcal capitalista las mujeres poseen el "poder oculto" que es la otra cara del "poder legítimo"»".

De igual forma, como se señala en el párrafo anterior, resulta importante indicar que las relaciones de poder establecidas en la sociedad patriarcal, conllevan una dependencia mutua entre las personas que participan en ellas; en este sentido, tal y como indica Villareal ${ }^{13}$, «(...) se ha planteado la dependencia vital de la mujer de los ingresos del esposo, pero no se plantea la dependencia -también vital- que tiene el hombre de los servicios que la mujer presta por medio del trabajo reproductivo». Este tipo de vínculo, en el cual están presentes «el poder masculino» y «el poder oculto», afecta negativamente tanto a mujeres como a hombres.

No obstante, en lo que respecta a las relaciones impropias sustentadas en una diferencia de edad entre un hombre adulto y una niña o adolescente, estas conllevan brechas de madurez, conocimiento, independencia, autoridad, entre otros, sustentadas en vínculos de dominio; además sobrelleva consecuencias importantes para las jóvenes menores de edad, dado que son relaciones denominadas «pseudo-afectivas», que constituyen la

8. León Camacho, «Propuesta educativa...», 2018.

9. Ibíd, 6

10. Villarreal Montoya, «Relaciones de poder», 2001.

11. Ibíd, 6 .

12. Ibíd, 8

13. Ibíd, 8-9. 
esencia de la violencia de género. Este tipo de relación amenaza el desarrollo de niñas y mujeres adolescentes, violenta sus derechos, afecta su educación formal, su salud física y emocional, lo que las expone a otras situaciones de vulnerabilidad y abuso.

Como refiere el UNFPA ${ }^{14}$, desde un enfoque de derecho, las uniones impropias resultan para las menores de edad, «(...) además de inválidas, inconvenientes, inadecuadas y extemporáneas para ellas; y desde una perspectiva género sensitiva, como una forma oculta y socialmente legitimada de violencia en este ámbito». De igual manera, se puede establecer que las relaciones impropias son vínculos de poder asimétricos y abusivos que atropellan los derechos humanos de las niñas y adolescentes.

Por lo anterior, resulta importante contar con la Ley 19337 que protege a niñas y adolescentes de situaciones de violencia de género asociadas a relaciones abusivas de poder.

\section{En qué consiste la ley de relaciones impropias}

El proyecto de Ley $19337^{15}$, referente a situaciones de violencia de género asociadas a relaciones abusivas, plantea una conceptualización del término uniones impropias como:

vínculos sustentados en relaciones desiguales de poder entre un hombre y una niña o adolescente mujer, en tanto desde un enfoque de derechos estos resultan, además de inválidos, inconvenientes, inadecuados y extemporáneos para ellas y, desde una perspectiva género sensitiva, se constituyen en una forma oculta y socialmente legitimada de violencia basada en género.

Este proyecto fue presentado en setiembre de 2014 e incluye datos de gran relevancia para identificar la situación de vulnerabilidad de las niñas y adolescentes para vivir experiencias de relaciones abusivas y desiguales, en el marco de la definición de uniones impropias. Se hace referencia al UNFPA, que en su informe «Uniones Impropias y Embarazo en la Niñez y la Adolescencia en Costa Rica», a partir de datos estadísticos basados en el X Censo de Población y VI de Vivienda del 2011, mostró que ${ }^{16}$ :

- El $8.6 \%$ de las niñas y las adolescentes mujeres entre los 12 y los 19 años había estado alguna vez en algún tipo de vínculo de convivencia conyugal (7 \% en «unión» y $1.3 \%$ casadas).

- Cerca del $98 \%$ de las niñas y adolescentes que declararon su estado conyugal en el marco de una unión, esta era con hombres mayores de edad.

14. Fondo de Población, Uniones Tempranas..., 6 .

15. Asamblea Legislativa de la República de Costa Rica, Proyecto de Ley 19.337, del 30 de setiembre, reforma de los artículos 159 y 161 de la Ley n. ${ }^{\circ} 4573$, Código Penal; reforma de los artículos 14, 16 y 158 y derogatoria de los artículos 21, 22, 36 y 38 de la Ley n. ${ }^{\circ}$ 5476, Código de Familia; reforma del artículo 39 de la Ley n. ${ }^{\circ}$ 63, Código Civil, y Reforma del artículo 89 de la Ley n. ${ }^{\circ}$ 3504, Ley orgánica del Tribunal Supremo de Elecciones y del Registro Civil, para el fortalecimiento de la protección legal de niñas y adolescentes mujeres, ante situaciones de violencia de género asociadas a relaciones abusivas, expediente $\mathrm{n}{ }^{\circ}$ 19.337, (2014): 1, acceso: 26 de marzo, 2020, http://www.asamblea.go.cr/sd/SiteAssets/Lists/Consultas $\% 20$ Biblioteca/EditForm/Proyecto-19337\%20relaciones\%20menores\%20de\%20edad.pdf

16. Ibíd, 2 . 
Otro punto de gran relevancia, considerado para la formulación de este proyecto de ley, fue el embarazo adolescente, ya que este se asocia a la denominada unión impropia ${ }^{17}$ :

«Con respecto del embarazo en la adolescencia los datos del informe reflejan que casi 9 de cada 100 niñas y adolescentes mujeres ha tenido al menos una hija o hijo nacido vivo. Finalmente, un $55 \%$ de las niñas y adolescentes madres vive en vínculo de convivencia».

Según el panorama expuesto anteriormente, y ante la obligación del Estado costarricense de garantizar el respeto a las personas menores de edad, según la Convención sobre los Derechos del Niño de 1990 y el Código de la Niñez y la Adolescencia de 1998; surge dicho proyecto de ley, el cual fue aprobado como la Ley 9406 para el fortalecimiento de la protección legal de las niñas y las adolescentes mujeres ante situaciones de violencia de género asociadas a relaciones abusivas, por la Asamblea Legislativa de la República de Costa Rica, el 30 de noviembre de 2016.

Es así como en el marco normativo del Estado costarricense, las relaciones impropias se definen a partir de lo estipulado en la Ley 9406, que aborda estos vínculos a partir de las diferencias de edades existentes entre la persona menor de edad y la persona mayor de edad.

Dentro de los delitos indicados en la Ley 9406, se sanciona con cárcel a la persona mayor que mantenga relaciones sexuales con personas menores de edad y abusos sexuales contra personas menores de edad y personas incapaces, en las condiciones que se detallan en los cuadros 1 y 2.

\section{CUADRO 1}

Tipificación del delito Relaciones sexuales con personas menores de edad, según Ley 9406

\begin{tabular}{|c|c|c|c|}
\hline \multicolumn{4}{|c|}{$\begin{array}{l}\text { Definición del delito: Relaciones sexuales con personas menores de edad } \\
\text { Quien se haga acceder o tenga acceso carnal por vía oral, anal o vaginal, } \\
\text { con una persona menor de edad, siempre que no constituya delito de violación }\end{array}$} \\
\hline $\begin{array}{l}\text { Cuando la persona } \\
\text { menor de edad sea: }\end{array}$ & $\begin{array}{l}\text { Cuando la persona } \\
\text { mayor de edad sea: }\end{array}$ & $\begin{array}{r}\text { Pena } \\
\text { Relaciones sexu }\end{array}$ & $\begin{array}{l}\text { pulada por el delito } \\
\text { con personas menores de edad }\end{array}$ \\
\hline $\begin{array}{l}\text { Mayor de trece } \\
\text { y menor de quince } \\
\text { años de edad }\end{array}$ & $\begin{array}{l}\text { Cinco o más años } \\
\text { mayor en edad }\end{array}$ & $\begin{array}{l}\text { Pena de prisión } \\
\text { de tres a seis años }\end{array}$ & $\begin{array}{l}\text { Pena de prisión de cuatro a diez años } \\
\text { en el caso de que el autor del delito } \\
\text { tenga una relación de parentesco de }\end{array}$ \\
\hline $\begin{array}{l}\text { Mayor de quince } \\
\text { y menor de } \\
\text { dieciocho años }\end{array}$ & $\begin{array}{l}\text { Siete o más años } \\
\text { mayor en edad }\end{array}$ & $\begin{array}{l}\text { Pena de prisión } \\
\text { de dos a tres años }\end{array}$ & $\begin{array}{l}\text { consanguinidad o afinidad, sea tutor, } \\
\text { o se encuentre en una posición de } \\
\text { confianza o autoridad con respecto } \\
\text { de la víctima o su familia }\end{array}$ \\
\hline
\end{tabular}

Fuente: Ley 9406 para el fortalecimiento de la protección legal de las niñas y las adolescentes mujeres ante situaciones de violencia de género asociadas a relaciones abusivas.

17. Ibíd, 2 . 


\section{CUADRO 2}

Tipificación del delito Abusos sexuales contra personas menores de edad y personas incapaces, según Ley 9406

\section{Definición del delito: Abusos sexuales contra personas menores de edad y personas incapaces:}

Cuando una persona mayor de edad cometa de manera abusiva actos con fines sexuales a la persona menor de edad o incapaz contra una persona menor de edad o incapaz o la obligue a realizarlos al agente, a sí misma o a otra persona, siempre que no constituya delito de violación.

Cuando la persona sea:

Pena estipulada por el delito Abusos sexuales contra personas menores de edad y personas incapaces

Menor de edad o incapaz o la obligue a realizarlos al agente, a sí misma o a otra persona, siempre que no

Pena será de tres a ocho años de prisión constituya delito de violación

Menor de edad o incapaz sea menor de quince años

Pena será de cuatro a diez años de prisión

Fuente: Ley 9406 para el fortalecimiento de la protección legal de las niñas y las adolescentes mujeres ante situaciones de violencia de género asociadas a relaciones abusivas.

Además, es necesario destacar que esta ley plantea una reforma a algunos artículos del Código de Familia, en el marco de la protección de los derechos de las personas menores de edad; principalmente demarcado en la imposibilidad del matrimonio con una persona menor de edad, así como la obligatoriedad del padre, madre o tutor de resguardar a la persona menor de edad ante una situación de riesgo tal como las uniones impropias.

\section{CUADRO 3}

Modificación realizada al Código de Familia a partir de la aprobación de Ley 9406

Artículo Modificación al Código de Familia

Artículo 14, Artículo 14. Es legalmente imposible el matrimonio:

incisos 4) y 7) 4) Entre quien adopta y la persona adoptada y sus descendientes; hijos e hijas adoptivos de la misma persona; la persona adoptada y los hijos e hijas de quien adopta; la persona adoptada y el excónyuge de quien adopta, y la persona que adopta y el excónyuge de quien es adoptado.

7) De la persona menor de dieciocho años.

Artículo 64 Artículo 64. La nulidad del matrimonio, prevista en el artículo 14 de esta ley, se declarará de oficio. El Registro Civil no inscribirá el matrimonio de las personas menores de dieciocho años.

Articulo 148 Artículo 148. Quien ejerza la patria potestad entregará a su hijo mayor o a la persona que lo reemplace en la administración, cuando esta concluya por otra causa, todos los bienes y frutos que pertenezcan al hijo y rendirá cuenta general de dicha administración.

Artículo 158 Artículo 158. Suspensión de la patria potestad

La patria potestad termina:

a) Por la mayoridad adquirida.

c) Por la declaratoria judicial de abandono, que se produzca por encontrarse la persona menor de edad en riesgo social, de acuerdo con el artículo 175 de este Código, y no exista oposición de los padres o cuando, suspendido el derecho, ellos no demuestren haber modificado la situación de riesgo para la persona menor de edad, en el plazo que el juez les haya otorgado.

d) Cuando la persona menor de edad haya sido objeto de violación, abuso sexual, corrupción o lesiones graves o gravísimas de quienes la ejerzan.

Fuente: Ley 9406 para el fortalecimiento de la protección legal de las niñas y las adolescentes mujeres ante situaciones de violencia de género asociadas a relaciones abusivas. 
Adicional a los cambios ya señalados, la Ley 9406, planteó la modificación del artículo número 89 de la Ley 3504, Ley Orgánica del Tribunal Supremo de Elecciones (TSE) y del Registro Civil, como se detalla a continuación:

\section{CUADRO 4}

Modificación a la Ley Orgánica del Tribunal Supremo de Elecciones (TSE) y del Registro Civil a partir de la aprobación de Ley 9406

\begin{tabular}{ll}
\hline Artículo & Modificación a la Ley Orgánica del Tribunal Supremo de Elecciones (TSE) y del Registro Civil \\
Artículo 89 & $\begin{array}{l}\text { Todo costarricense de uno u otro sexo, mayor de dieciocho años, tiene la obligación ineludible de } \\
\text { adquirir su cédula de identidad. }\end{array}$
\end{tabular}

Fuente: Ley 9406 para el fortalecimiento de la protección legal de las niñas y las adolescentes mujeres ante situaciones de violencia de género asociadas a relaciones abusivas.

Es así como la Ley 9406 ha sido aprobada por la Asamblea Legislativa para su acatamiento como medida para la protección de las personas menores de edad, principalmente de las niñas y las adolescentes, población en mayor condición de vulnerabilidad frente a este fenómeno social. Sin embargo, es necesario destacar que esta ley deberá de ir acompañada de un proceso de conciencia social sobre las implicaciones que conlleva este tipo de relaciones para las personas menores de edad y, por consiguiente, la necesidad de denunciar estos delitos como medida de atención de los casos de violencia desde la población.

\section{Alcance de las estadísticas de nacimientos en Costa Rica} en el marco de relaciones impropias, periodo 2000-2018

De acuerdo con la base de datos sobre nacimientos del INEC, se puede identificar un total de 22238 casos de relaciones impropias durante el periodo 2000-2018, esto corresponde a casi un $20 \%$ del total de los nacimientos registrados en dicho periodo para las madres entre 13 y 17 años. Como también se puede observar en la tabla 1, la mayor cantidad de estos casos corresponden a madres adolescentes entre los 16 y 17 años, con un porcentaje del $73 \%$. Asimismo, se puede apreciar como la frecuencia de casos totales de relaciones impropias viene en descenso, pasando de más de 1556 casos en el 2000 a 332 en el 2018. 
TABLA 1

Distribución absoluta de nacimientos en el marco de relaciones impropias, según edad de las madres de 13 a 17 años durante el periodo 2000-2018

\begin{tabular}{|c|c|c|c|c|c|c|}
\hline Año & $\begin{array}{l}\text { Madre } 13 \text { años y } \\
\text { padre de } 18 \text { años } \\
\text { o más }\end{array}$ & $\begin{array}{l}\text { Madre } 14 \text { años y } \\
\text { padre de } 19 \text { años } \\
\text { o más }\end{array}$ & $\begin{array}{l}\text { Madre } 15 \text { años y } \\
\text { padre de } 22 \text { años } \\
\text { o más }\end{array}$ & $\begin{array}{l}\text { Madre } 16 \text { años y } \\
\text { padre de } 23 \text { años } \\
\text { o más }\end{array}$ & $\begin{array}{l}\text { Madre } 17 \text { años y } \\
\text { padre de } 24 \text { años } \\
\text { o más }\end{array}$ & Total \\
\hline 2000 & 23 & 156 & 280 & 420 & 677 & 1556 \\
\hline 2001 & 28 & 159 & 259 & 500 & 588 & 1534 \\
\hline 2002 & 22 & 147 & 248 & 473 & 705 & 1595 \\
\hline 2003 & 29 & 126 & 253 & 494 & 706 & 1608 \\
\hline 2004 & 33 & 119 & 238 & 446 & 682 & 1518 \\
\hline 2005 & 27 & 130 & 278 & 469 & 619 & 1523 \\
\hline 2006 & 22 & 163 & 234 & 484 & 612 & 1515 \\
\hline 2007 & 31 & 140 & 252 & 419 & 586 & 1428 \\
\hline 2008 & 33 & 117 & 214 & 390 & 545 & 1299 \\
\hline 2009 & 24 & 104 & 215 & 375 & 542 & 1260 \\
\hline 2010 & 16 & 94 & 187 & 320 & 431 & 1048 \\
\hline 2011 & 25 & 93 & 187 & 337 & 439 & 1081 \\
\hline 2012 & 18 & 98 & 183 & 312 & 494 & 1105 \\
\hline 2013 & 19 & 97 & 207 & 310 & 409 & 1042 \\
\hline 2014 & 14 & 85 & 156 & 275 & 385 & 915 \\
\hline 2015 & 5 & 75 & 130 & 262 & 364 & 836 \\
\hline 2016 & 10 & 46 & 117 & 215 & 302 & 690 \\
\hline 2017 & 3 & 24 & 52 & 150 & 224 & 453 \\
\hline 2018 & 1 & 23 & 43 & 80 & 185 & 332 \\
\hline Total & 383 & 1996 & 3733 & 6731 & 9495 & 22338 \\
\hline $\begin{array}{l}\text { Nacimientos } \\
\text { en esa edad }\end{array}$ & 1517 & 7046 & 19265 & 36210 & 52436 & 116474 \\
\hline Porcentaje & $25,2 \%$ & $28,3 \%$ & $19,4 \%$ & $18,6 \%$ & $18,1 \%$ & $19,2 \%$ \\
\hline
\end{tabular}

Fuente: Elaboración propia, basado en Estadísticas de nacimientos, INEC, procesados a través de REDATAM 7.

Si esta información se examina a nivel geográfico, las provincias de San José, Alajuela, Puntarenas y Limón son las que más casos registran. Por su parte, Heredia, Cartago y Guanacaste son las que registran menos caos. No obstante, se debe prestar atención a los nacimientos en madres de 13 y 14 años en las provincias de Puntarenas y Limón, en donde la cantidad de relaciones impropias es bastante superior con respecto de las otras provincias que se ubican fuera el Valle Central que tienen una mayor densidad poblacional. 
TABLA 2

Distribución absoluta de relaciones impropias por provincia, según registro de nacimientos en madres de 13 a 17 años durante el periodo 2000-2018

\begin{tabular}{|c|c|c|c|c|c|c|c|}
\hline Provincia & San José & Alajuela & Cartago & Heredia & Guanacaste & Puntarenas & Limón \\
\hline $\begin{array}{l}\text { Madre } 13 \text { años y padre } \\
\text { de } 18 \text { años o más }\end{array}$ & 59 & 68 & 34 & 16 & 33 & 90 & 83 \\
\hline $\begin{array}{l}\text { Madre } 14 \text { años y padre } \\
\text { de } 19 \text { años o más }\end{array}$ & 410 & 419 & 170 & 84 & 150 & 404 & 359 \\
\hline $\begin{array}{l}\text { Madre } 15 \text { años y padre } \\
\text { de } 22 \text { años o más }\end{array}$ & 811 & 764 & 316 & 170 & 380 & 730 & 562 \\
\hline $\begin{array}{l}\text { Madre } 16 \text { años y padre } \\
\text { de } 23 \text { años o más }\end{array}$ & 1608 & 1482 & 564 & 317 & 653 & 1193 & 914 \\
\hline $\begin{array}{l}\text { Madre } 17 \text { años y padre } \\
\text { de } 24 \text { años o más }\end{array}$ & 2286 & 2159 & 810 & 483 & 885 & 1604 & 1268 \\
\hline Total & 5174 & 4892 & 1894 & 1070 & 2101 & 4021 & 3186 \\
\hline
\end{tabular}

Fuente: Elaboración propia, basado en Estadísticas de nacimientos, INEC, procesados a través de REDATAM 7.

Si se enlistan los 15 cantones con más y menos casos, los que más registran a lo largo de todo el periodo, corresponde a San José, San Carlos y Alajuela, Desamparados, Puntarenas, Pococí y Limón. Por su parte, San Mateo, Dota, Turrubares, San Pablo, León Cortés y Tarrazú son los cantones que menos casos registran.

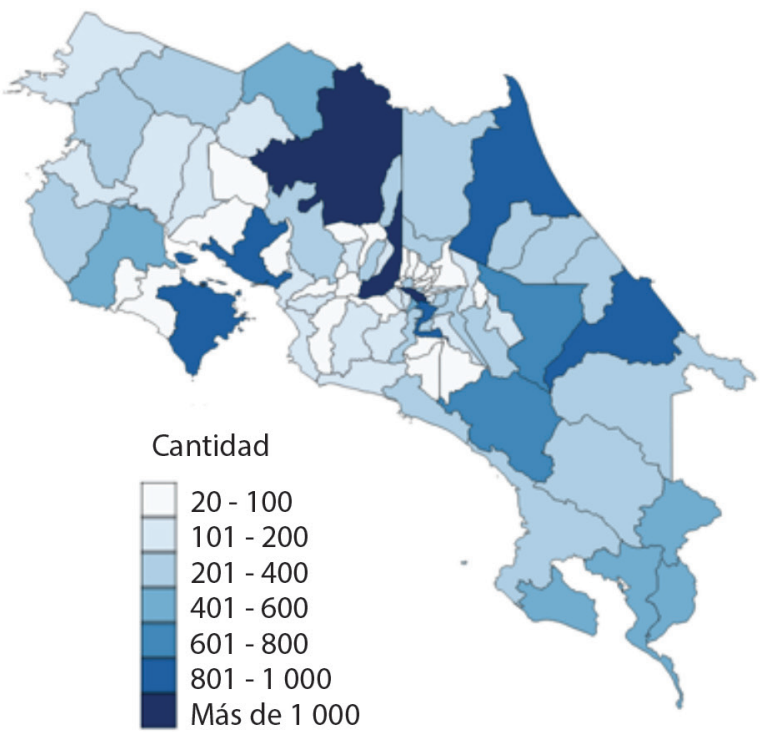

\begin{tabular}{|lc|l|c|}
\hline \multicolumn{2}{|c|}{ Cantones con más casos } & \multicolumn{2}{|c|}{ Cantones con menos casos } \\
\hline San José & 1368 & San Mateo & 20 \\
\hline San Carlos & 1329 & Dota & 21 \\
\hline Alajuela & 1231 & Turrubares & 31 \\
\hline Desamparados & 980 & San Pablo & 31 \\
\hline Puntarenas & 975 & León Cortes & 32 \\
\hline Pococí & 961 & Tarrazú & 38 \\
\hline Limón & 852 & Alvarado & 40 \\
\hline Pérez Zeledón & 621 & Zarcero & 42 \\
\hline Turrialba & 608 & San Isidro & 42 \\
\hline Golfito & 569 & Belén & 43 \\
\hline Corredores & 535 & Flores & 46 \\
\hline Coto Brus & 459 & Hojancha & 49 \\
\hline Alajuelita & 429 & Montes de Oro & 54 \\
\hline Los Chiles & 414 & Montes de Oca & 55 \\
\hline Nicoya & 401 & Barva & 63 \\
\hline
\end{tabular}

Mapa 1. Distribución absoluta de relaciones impropias por cantón, según registro de nacimientos en madres de 13 a 17 años durante el periodo 2000-2018. Fuente: Elaboración propia, basado en Estadísticas de nacimientos, INEC, procesados a través de REDATAM 7. 
Con respecto de la edad de los padres al momento del nacimiento, en su mayoría no superan los 30 años. No obstante, se presentan casos en que los padres superan los 40 años, incluso se reportan edades mayores a los 60 años. No obstante, uno de los aspectos que llama la atención es la cantidad de casos de padres en los cuales se ignora su edad o no son declarados al momento del nacimiento. Como se muestra en la tabla 3, estas dos situaciones son más frecuentes en las madres de 13 y 14 años, en los cuales el porcentaje supera el $60 \%$ de los casos a lo largo del periodo estudiado.

\section{TABLA 3}

Edad de los padres identificados en una relación impropia, según registro de nacimientos en madres de 13 a 17 años durante el periodo 2000-2018

\begin{tabular}{lccccc} 
& \multicolumn{5}{c}{ Edad de la madre } \\
\hline Edad del padre & 13 años & 14 años & 15 años & 16 años & 17 años \\
Menos de 30 años & 406 & 2461 & 7728 & 15807 & 23888 \\
De 30 a 39 años & 30 & 189 & 542 & 1249 & 2279 \\
De 40 a 49 años & 10 & 30 & 142 & 247 & 499 \\
50 años o más & 9 & 10 & 51 & 74 & 121 \\
Padre no declarado & 598 & 1939 & 4107 & 6528 & 8399 \\
Edad ignorada & 464 & 2417 & 6695 & 12305 & 17250 \\
Total & 1517 & 7046 & 19265 & 36210 & 52436 \\
Porcentaje de padres con & $70 \%$ & $61,8 \%$ & $56,1 \%$ & $52 \%$ & $48,9 \%$ \\
edad ignorada o no declarado & & &
\end{tabular}

Fuente: Elaboración propia, basado en Estadísticas de nacimientos, INEC, procesados a través de REDATAM 7.

Por otra parte, si se examina la nacionalidad de las madres niñas y adolescentes identificadas en una relación impropia durante el periodo en estudio, en su mayoría son costarricenses, pero resulta importante destacar el porcentaje de madres de nacionalidad nicaragüense, que representa alrededor del $15 \%$ de los casos ocurridos en el periodo.

TABLA 4

Porcentaje de madres niñas y adolescentes nicaragüenses identificadas en una relación impropia durante el periodo 2000-2018

\begin{tabular}{cccccc} 
Año & $\begin{array}{c}\text { Madre 13 años } \\
\text { y padre de 18 años } \\
\text { o más }\end{array}$ & $\begin{array}{c}\text { Madre 14 años } \\
\text { y padre de 19 años } \\
\text { o más }\end{array}$ & $\begin{array}{c}\text { Madre 15 años } \\
\text { y padre de 22 años } \\
\text { o más }\end{array}$ & $\begin{array}{c}\text { Madre 16 años } \\
\text { y padre de 23 años } \\
\text { o más }\end{array}$ & $\begin{array}{c}\text { Madre 17 años } \\
\text { y padre de 24 años } \\
\text { o más }\end{array}$ \\
\hline 2000 & $8,7 \%$ & $13,5 \%$ & $16,1 \%$ & $17,9 \%$ & $16,1 \%$ \\
2001 & $7,1 \%$ & $17 \%$ & $18,1 \%$ & $20,2 \%$ & $17,9 \%$ \\
2002 & $18,2 \%$ & $14,3 \%$ & $21 \%$ & $14,4 \%$ & $16,9 \%$ \\
2003 & $10,3 \%$ & $24,6 \%$ & $18,2 \%$ & $14,2 \%$ & $14,2 \%$ \\
2004 & $12,1 \%$ & $16,8 \%$ & $18,1 \%$ & $13,7 \%$ & $14,7 \%$ \\
\hline
\end{tabular}




\begin{tabular}{cccccc} 
Año & $\begin{array}{c}\text { Madre 13 años } \\
\text { y padre de 18 años } \\
\text { o más }\end{array}$ & $\begin{array}{c}\text { Madre } 14 \text { años } \\
\text { y padre de } 19 \text { años } \\
\text { o más }\end{array}$ & $\begin{array}{c}\text { Madre } 15 \text { años } \\
\text { y padre de } 22 \text { años } \\
\text { o más }\end{array}$ & $\begin{array}{c}\text { Madre } 16 \text { años } \\
\text { y padre de } 23 \text { años } \\
\text { o más }\end{array}$ & $\begin{array}{c}\text { Madre } 17 \text { años } \\
\text { y padre de } 24 \text { años } \\
\text { o más }\end{array}$ \\
2005 & $22,2 \%$ & $20 \%$ & $16,2 \%$ & $14,3 \%$ & $16,6 \%$ \\
2006 & $31,8 \%$ & $20,9 \%$ & $17,5 \%$ & $20,2 \%$ & $16,3 \%$ \\
2007 & $22,6 \%$ & $17,1 \%$ & $19,4 \%$ & $15,8 \%$ & $16 \%$ \\
2008 & $18,2 \%$ & $17,1 \%$ & $14,5 \%$ & $15,9 \%$ & $17,4 \%$ \\
2009 & $8,3 \%$ & $16,3 \%$ & $17,7 \%$ & $16,8 \%$ & $18,1 \%$ \\
2010 & $12,5 \%$ & $9,6 \%$ & $17,6 \%$ & $16,9 \%$ & $19,3 \%$ \\
2011 & $16 \%$ & $12,9 \%$ & $15 \%$ & $16 \%$ & $13,7 \%$ \\
2012 & $16,7 \%$ & $15,3 \%$ & $13,1 \%$ & $11,9 \%$ & $17 \%$ \\
2013 & $0,0 \%$ & $14,4 \%$ & $12,6 \%$ & $13,5 \%$ & $15,9 \%$ \\
2014 & $7,1 \%$ & $15,3 \%$ & $14,1 \%$ & $17,1 \%$ & $11,7 \%$ \\
2015 & $0,0 \%$ & $8 \%$ & $15,4 \%$ & $10,7 \%$ & $14 \%$ \\
2016 & $10 \%$ & $6,5 \%$ & $7,7 \%$ & $19,1 \%$ & $19,2 \%$ \\
2017 & $0,0 \%$ & $8,3 \%$ & $11,5 \%$ & $9,3 \%$ & $15,2 \%$ \\
2018 & $0,0 \%$ & $8,7 \%$ & $11,6 \%$ & $16,3 \%$ & $20,5 \%$ \\
Total & $\mathbf{1 4 , 1 \%}$ & $\mathbf{1 5 , 9} \%$ & $\mathbf{1 6 , 3} \%$ & $\mathbf{1 5 , 8} \%$ & $\mathbf{1 6 , 2} \%$ \\
\hline
\end{tabular}

Fuente: Elaboración propia, basado en Estadísticas de nacimientos, INEC, procesados a través de REDATAM 7.

Es oportuno señalar que, durante el periodo de estudio, se registran casos de madres niñas y adolescentes nicaragüenses cuyos padres son tanto nicaragüenses como costarricenses. De igual forma, se registran casos de niñas y adolescentes que se convirtieron en madres, de nacionalidad costarricense, con padres nicaragüenses.

TABLA 5

Distribución absoluta de relaciones impropias por nacionalidad de la madre y del padre, según registro de nacimientos en madres de 13 a 17 años durante el periodo 2000-2018

\begin{tabular}{|c|c|c|c|c|c|}
\hline \multirow{2}{*}{$\begin{array}{c}\text { Nacionalidad de la madre } \\
\text { Nicaragüense }\end{array}$} & \multicolumn{2}{|c|}{ Nacionalidad del padre } & \multirow{2}{*}{$\begin{array}{c}\text { Nacionalidad de la madre } \\
\text { Costarricense }\end{array}$} & \multicolumn{2}{|c|}{ Nacionalidad del padre } \\
\hline & Costarricense & Nicaragüense & & Costarricense & Nicaragüense \\
\hline $\begin{array}{l}\text { Madre } 13 \text { años y } \\
\text { padre de } 18 \text { años o más }\end{array}$ & 19 & 26 & $\begin{array}{l}\text { Madre } 13 \text { años y } \\
\text { padre de } 18 \text { años o más }\end{array}$ & 243 & 30 \\
\hline $\begin{array}{l}\text { Madre } 14 \text { años y } \\
\text { padre de } 19 \text { años o más }\end{array}$ & 83 & 160 & $\begin{array}{l}\text { Madre } 14 \text { años y } \\
\text { padre de } 19 \text { años o más }\end{array}$ & 1241 & 144 \\
\hline $\begin{array}{l}\text { Madre } 15 \text { años y } \\
\text { padre de } 22 \text { años o más }\end{array}$ & 189 & 293 & $\begin{array}{l}\text { Madre } 15 \text { años y } \\
\text { padre de } 22 \text { años o más }\end{array}$ & 2344 & 271 \\
\hline $\begin{array}{l}\text { Madre } 16 \text { años y } \\
\text { padre de } 23 \text { años o más }\end{array}$ & 316 & 498 & $\begin{array}{l}\text { Madre } 16 \text { años y } \\
\text { padre de } 23 \text { años o más }\end{array}$ & 4426 & 430 \\
\hline $\begin{array}{l}\text { Madre } 17 \text { años y } \\
\text { padre de } 24 \text { años o más }\end{array}$ & 512 & 700 & $\begin{array}{l}\text { Madre } 17 \text { años y } \\
\text { padre de } 24 \text { años o más }\end{array}$ & 6324 & 470 \\
\hline
\end{tabular}

Fuente: Elaboración propia, basado en Estadísticas de nacimientos, INEC, procesados a través de REDATAM 7. 


\section{Alcance de las estadísticas de matrimonios en Costa Rica}

en el marco de relaciones impropias, periodo 2000-2018

Según el Código de Familia de Costa Rica, en el artículo 11: «El matrimonio es la base esencial de la familia y tiene por objeto la vida en común, la cooperación y el mutuo auxilio». El matrimonio es concebido como la manifestación de las personas contrayentes a través del consentimiento legal y expreso de la unión.

El Código de Familia ha tenido diversas modificaciones, en el marco de las uniones con personas menores de edad; primeramente en febrero de 2007, para impedir el matrimonio de menores de 15 años, esto mediante la reforma 8571, de los artículos 14 y 64 del Código de Familia, además el artículo 38 del Código Civil, el artículo 181 del Código Penal y se da la derogación del inciso 3) del artículo 15, el artículo 19 y el inciso c) del artículo 65 del Código de Familia.

Por lo cual, al analizar los datos de declaración de matrimonios, donde la cónyuge corresponde a una mujer menor de edad, durante el periodo 2000-2018, se identifica la inscripción de uniones de niñas y adolescentes menores de 15 años durante el periodo 2000-2007, ya que para el momento señalado no existía impedimento alguno para llevar a cabo matrimonios con personas menores de 18 años, tal como se detalla en la tabla 6.

TABLA 6

Distribución absoluta de matrimonios con niñas y adolescentes, durante el periodo 2000-2018

\begin{tabular}{cc} 
Edad de la niña/adolescente & Matrimonios 2000-2018 \\
10 & 1 \\
12 & 6 \\
13 & 61 \\
14 & 330 \\
15 & 2708 \\
16 & 5086 \\
17 & 7776 \\
Total & $\mathbf{1 5 9 6 8}$ \\
\hline
\end{tabular}

Fuente: Elaboración propia, basado en Estadísticas de matrimonios, INEC, procesados a través de REDATAM 7.

Adicional a lo señalado, se llevó a cabo una segunda modificación al Código de Familia, en concordancia con lo estipulado en la Ley 9406, la cual entró a regir a partir de enero de 2017, y que establece, en Costa Rica, la imposibilidad de celebrar e inscribir matrimonios cuando una de las personas contrayentes sea menor de edad.

De la totalidad de matrimonios ocurridos en el periodo de estudio, 2000-2018, correspondiente a 15 968, se destaca que 6407 (40,1\%) corresponden a matrimonios declarados entre un hombre mayor de edad y una niña o adolescente; cabe recalcar que el cálculo realizado contempló los rangos de diferencia de edades de cinco y siete años entre ambos conyugues, según determina la Ley 9406 en su definición operativa para identificar las relaciones impropias. 


\section{TABLA 7}

Distribución absoluta de los matrimonios de niñas y adolescentes, ocurridos en Costa Rica en el periodo 2000-2018, según la identificación de relaciones impropias de acuerdo con rangos de edad estipulados en la Ley 9406

\begin{tabular}{cc} 
Edad de la niña/adolescente & Matrimonios 2000-2018 \\
10 & 1 \\
12 & 6 \\
13 & 60 \\
14 & 326 \\
15 & 1276 \\
16 & 2013 \\
17 & 2725 \\
Total & $\mathbf{6 4 0 7}$ \\
\hline
\end{tabular}

Fuente: Elaboración propia, basado en Estadisticas de matrimonios, INEC, procesados a través de REDATAM 7.

Al hacer una revisión de la tendencia de inscripción de matrimonios entre niñas o adolescentes con hombres mayores de edad, en el marco de relaciones impropias, en el periodo de estudio 2000-2018, se observa un decrecimiento en el reporte de las estadísticas de los casos identificados.

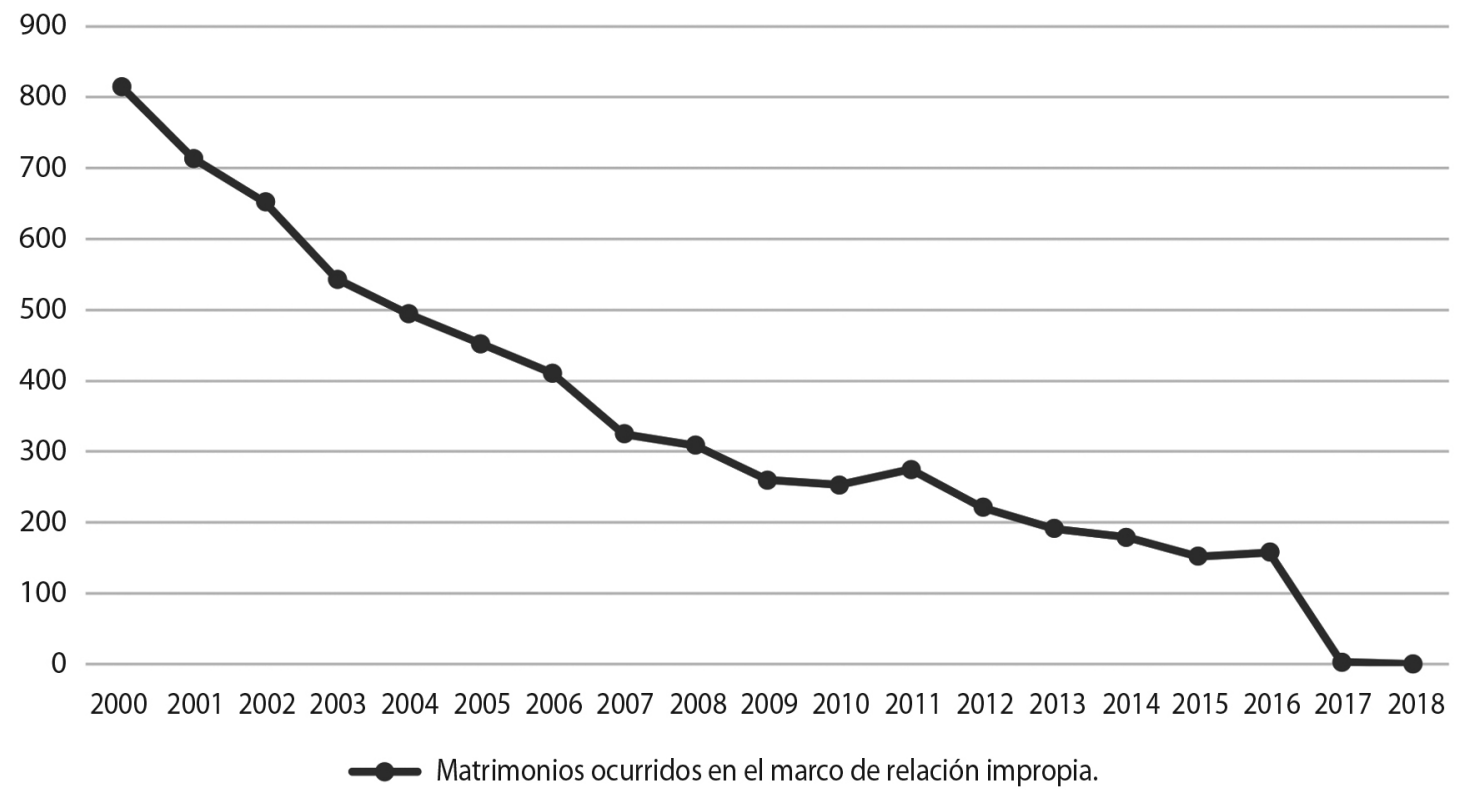

Gráfico 1. Tendencia de inscripciones de matrimonios de niñas y adolescentes durante el periodo 2000-2018, según identificación de relaciones impropias en Costa Rica, de acuerdo con rangos de edad estipulados en la Ley 9406. Fuente: Elaboración propia, basado en Estadisticas de matrimonios, INEC, procesados a través de REDATAM 7. 
De acuerdo con la tabla 8, a partir de 2000, punto en el que se alcanzó el máximo del periodo en estudio con 815 casos, se disminuye de forma significativa el número de matrimonios inscritos, a partir de los casos identificados como relaciones impropias, lo que podría asociarse parcialmente a cambios efectuados en el marco jurídico nacional, ocurrido en el 2007, en el cual descendió a 325 casos y en el 2017, en el cual descendió a 3 casos, hasta llegar a 0 inscripciones en el 2018. Estos hechos son concordantes con lo estipulado en la reforma 8571 al Código de Familia y la Ley 9406.

TABLA 8

Matrimonios de niñas y adolescentes ocurridos en Costa Rica

en el marco de relación impropia, según año de declaración

\begin{tabular}{lc} 
Año & Matrimonios ocurridos en el marco de relación impropia \\
2000 & 815 \\
2001 & 714 \\
2002 & 652 \\
2003 & 543 \\
2004 & 494 \\
2005 & 452 \\
2006 & 411 \\
2007 & 325 \\
2008 & 309 \\
2009 & 260 \\
2010 & 253 \\
2011 & 275 \\
2012 & 221 \\
2013 & 191 \\
2014 & 179 \\
2015 & 152 \\
2016 & 158 \\
2017 & 3 \\
2018 & 0 \\
Total & 6407 \\
\hline
\end{tabular}

Fuente: Elaboración propia, basado en Estadísticas de matrimonios, INEC, procesados a través de REDATAM 7.

Finalmente, resulta de interés observar el comportamiento de estas uniones a nivel geográfico, para identificar las provincias y los cantones que presentaron un mayor número de inscripciones de matrimonios durante el periodo de estudio 2000-2018. 


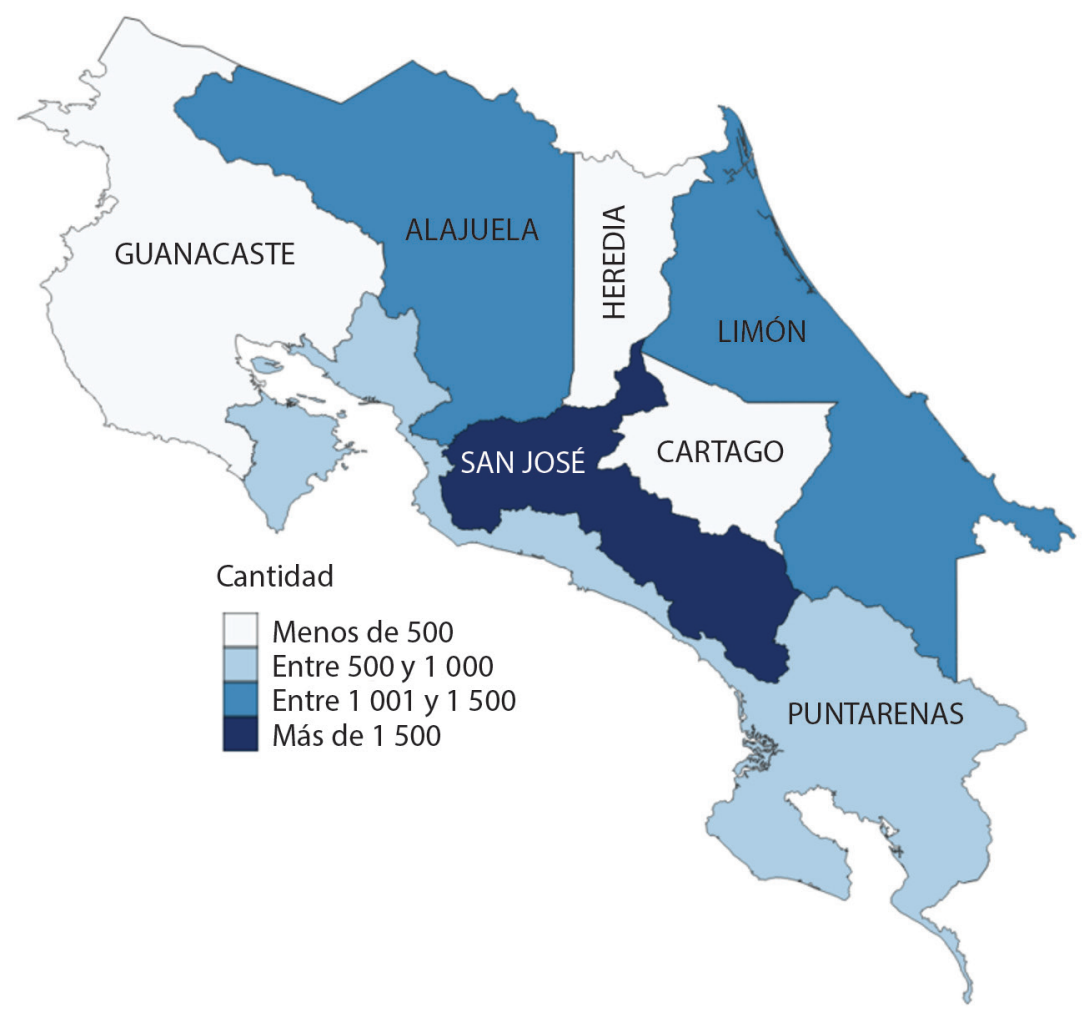

Mapa 2. Matrimonios de niñas y adolescentes ocurridos en Costa Rica durante el periodo 2000-2018, en el marco de relación impropia, según provincia de declaración. Fuente: Elaboración propia, basado en Estadísticas de matrimonios, INEC, procesados a través de REDATAM 7.

A partir de lo observado en el mapa anterior, se identifica que San José (1766) se mantiene como la provincia de mayor declaración de la inscripción de matrimonios durante el periodo de estudio 2000-2018, seguido por Alajuela (1317), Limón (1020) y Puntarenas (930).

TABLA 9

Matrimonios de niñas y adolescentes ocurridos en Costa Rica en el marco de relación impropia, según provincia y año de declaración

\begin{tabular}{ccccccccc} 
Año & San José & Alajuela & Cartago & Heredia & Guanacaste & Puntarenas & Limón & Total \\
2000 & 251 & 162 & 59 & 57 & 55 & 102 & 129 & 815 \\
2001 & 211 & 138 & 51 & 41 & 50 & 115 & 108 & 714 \\
2002 & 209 & 131 & 59 & 39 & 52 & 89 & 73 & 652 \\
2003 & 127 & 123 & 54 & 32 & 46 & 80 & 81 & 543 \\
2004 & 148 & 101 & 37 & 48 & 37 & 59 & 64 & 494 \\
2005 & 137 & 95 & 32 & 32 & 33 & 67 & 56 & 452 \\
2006 & 129 & 78 & 33 & 33 & 31 & 55 & 52 & 411 \\
\hline
\end{tabular}




\begin{tabular}{|c|c|c|c|c|c|c|c|c|}
\hline Año & San José & Alajuela & Cartago & Heredia & Guanacaste & Puntarenas & Limón & Total \\
\hline 2007 & 75 & 70 & 23 & 22 & 27 & 50 & 58 & 325 \\
\hline 2008 & 85 & 64 & 23 & 20 & 22 & 47 & 48 & 309 \\
\hline 2009 & 62 & 47 & 14 & 15 & 25 & 51 & 46 & 260 \\
\hline 2010 & 66 & 55 & 18 & 19 & 15 & 35 & 45 & 253 \\
\hline 2011 & 69 & 57 & 17 & 20 & 15 & 52 & 45 & 275 \\
\hline 2012 & 47 & 56 & 11 & 11 & 18 & 40 & 38 & 221 \\
\hline 2013 & 43 & 48 & 8 & 10 & 15 & 28 & 39 & 191 \\
\hline 2014 & 42 & 41 & 7 & 15 & 19 & 16 & 39 & 179 \\
\hline 2015 & 43 & 25 & 10 & 8 & 13 & 22 & 31 & 152 \\
\hline 2016 & 21 & 26 & 7 & 11 & 4 & 22 & 67 & 158 \\
\hline 2017 & 1 & 0 & 1 & 0 & 0 & 0 & 1 & 3 \\
\hline 2018 & 0 & 0 & 0 & 0 & 0 & 0 & 0 & 0 \\
\hline Total & 1766 & 1317 & 464 & 433 & 477 & 930 & 1020 & 6407 \\
\hline
\end{tabular}

Fuente: Elaboración propia, basado en Estadísticas de matrimonios, INEC, procesados a través de REDATAM 7.

Las reformas realizadas al Código de Familia, mencionadas anteriormente, han llevado a que, a partir del 2018, el registro de matrimonios con personas menores de edad no sea tramitado por el Registro Civil, razón por la cual estas uniones podrían estar migrando a unión de hecho ante la imposibilidad de contraer matrimonio; razón por la cual, a pesar de no registrar casos de 2018, no se quiere decir que este tipo de relaciones no siga presente en la realidad social de Costa Rica. Ante esto, sigue siendo relevante prestar especial atención en los esfuerzos de identificación y denuncia de los casos de relaciones impropias en favor de la protección de las personas menores de edad.

\section{Alcance de las estadísticas de denuncias por delitos vinculados a la Ley 9406}

En el marco de las reformas señaladas en la Ley 9406, se observa un aumento significativo en el crecimiento de los casos interpuestos ante el Ministerio Público, por delitos como: abuso sexual contra personas menores de edad e incapaces (sic.), y relaciones sexuales con personas menores de edad.

TABLA 10

Casos entrados netos en el Ministerio Público (penal adultos)

\begin{tabular}{ccc} 
Año & $\begin{array}{c}\text { Abusos sexuales contra personas menores } \\
\text { de edad e incapaces }\end{array}$ & $\begin{array}{c}\text { Relaciones sexuales con } \\
\text { personas menores de edad }\end{array}$ \\
2004 & 1708 & 317 \\
2005 & 1980 & 317 \\
2006 & 1805 & 295 \\
\hline
\end{tabular}




\begin{tabular}{ccc} 
Año & $\begin{array}{c}\text { Abusos sexuales contra personas menores } \\
\text { de edad e incapaces }\end{array}$ & $\begin{array}{c}\text { Relaciones sexuales con } \\
\text { personas menores de edad }\end{array}$ \\
2007 & 1909 & 441 \\
2008 & 2072 & 589 \\
2009 & 2380 & 720 \\
2010 & 2739 & 568 \\
2011 & 2641 & 702 \\
2012 & 2606 & 798 \\
2013 & 2956 & 977 \\
2014 & 2782 & 914 \\
2015 & 2623 & 938 \\
2016 & 2826 & 1067 \\
2017 & 3598 & 2220 \\
2018 & 3734 & 4034 \\
\hline
\end{tabular}

Fuente: Elaboración propia, basada en estadísticas del Observatorio de la violencia de género contra las mujeres y acceso a la justicia, Poder Judicial, Costa Rica, 2004-2018.

Es importante señalar que las estadísticas públicas disponibles en el Observatorio de violencia de género contra las mujeres y acceso a la justicia del Poder Judicial, reflejan un incremento de $218,61 \%$ entre el 2004 y el 2018 para el primer tipo de delito mencionado anteriormente, mientras que para el segundo tipo de delito se observa un crecimiento de $1272,5 \%$.

El aumento de denuncias y, por ende, de casos netos en el Ministerio Público, evidencia el reconocimiento, primeramente del Estado, y además de la población, en la cual estas conductas se observan como hechos delictivos, en tanto parece haber una mayor conciencia, conocimiento y aplicación de la ley, principalmente reflejada en el incremento significativo de casos en 2017 y 2018, a raíz de la aprobación de la Ley 9406.

\section{Conclusiones}

En el marco del análisis que se ha llevado a cabo durante el periodo de estudio acerca de las relaciones impropias en Costa Rica, se concluyen distintos aspectos que favorecen la comprensión del fenómeno y podrían enriquecer futuros estudios para ampliar la investigación sobre esta temática.

El sistema patriarcal ha naturalizado las relaciones impropias, por lo que se requiere un cambio en la estructura, en el tanto es importante crear conciencia para llevar a cabo la denuncia y sanción social de los casos de relaciones impropias, con el fin de proteger los derechos de las niñas y adolescentes, principalmente.

En el caso de las estadísticas de nacimientos, el análisis se realiza únicamente con los datos declarados que pueden ser consignados como relación impropia, pero persisten 
casos en los que se declara desconocida la edad del padre, o se indica padre desconocido, lo cual genera un subregistro de los casos.

En lo que respecta a las estadísticas de matrimonios, se evidencia que, a partir de la aprobación de la Ley 9406, disminuye a cero casos; sin embargo, esto podría implicar que las relaciones impropias se estén dando en el marco de uniones de hecho, lo que sería más complejo de registrar a nivel de las estadísticas de población.

La Ley 9406 se crea en función de la protección de las niñas y adolescentes, pero parece que la estructura patriarcal busca estrategias para «saltarse la ley», en el marco de lo que podría denominarse como una forma de protección al agresor, a través de la perpetuación de naturalizar este tipo de uniones; además, a través de la no denuncia del delito u omisión que permite que estos agresores no reciban la sanción legal correspondiente a este delito.

Con los cambios en la Ley 9406, se presenta un aumento significativo en las denuncias de casos de relaciones impropias; sin embargo, es necesario fortalecer la conciencia de esta forma de violencia contra las niñas y adolescentes, ya que no es percibido de esta forma por la totalidad de la población costarricense.

\section{ABSTRACT}

The one who lies with girls... denounced wakes up. Characterization of inappropriate relationships in Costa Rica during the period of 2000-2018

Law 9406 was approved in Costa Rica in November 2016 and it is known as the «Law of inappropriate relationships» which are defined by a link of partnership that is evidenced by the huge age differences among its members. From this point, a revision of sources of information was carried out such as the statistics of births and marriages obtained from the system of indicators of Instituto Nacional de Estadística y Censos (INEC), through REDATAM 7, representing an analysis of data available for the period 2000-2018.

This article talks about the phenomenon from two perspectives: unions through marriage and teen pregnancies. In both cases, by filtering the available information from the identification of an inappropriate relationship, it is possible to obtain the characterization of the behaviour during the course of the study. This shows that once political measures take place, it is feasible to influence on the populations' statistics. However, it is important to highlight that the statistics could present limitations because the coverage of the collected data about this phenomenon could be concealed or overlooked as a protective action toward the adolescents when they are making their declarations.

Key words: Inappropriate relationships, teen pregnancy, abuse of power, statistics of teen population, law against inappropriate relationships.

\section{RÉSUMÉ}

Celui qui couche avec des filles mineures... se lève à l'aube dénoncé. Caractérisation des relations illicites au Costa Rica dans la période 2000-2018

La loi 9406 a été adoptée au Costa Rica en novembre 2016; cette loi est connue comme la «Loi des relations inconvenantes» lesquelles sont définies par un lien de couple caractérisé par une grande différence d'âge entre ses membres. C'est à partir de cela qu'on a mené une révision des 
sources d'information telles que les statistiques des naissances et des mariages qui ont été obtenues du système de consultation d'indicateurs de l'Instituto Nacional de Estadística y Censos - INEC (Institut National de de la Statique et du Recensement) à travers de REDATAM 7. Ceci représente une analyse des données disponibles pour la période 2000-2018.

Cet article aborde le phénomène, de deux perspectives: les unions par mariage et les grossesses chez les adolescentes. Dans les deux cas, lorsque l'information disponible est filtrée par l'identification d'une relation illicite, il est possible de caractériser le comportement pendant la période d'étude. Cela met en évidence que quand les mesures politiques sont appliquées, il est possible d'avoir une incidence dans les statistiques de la population. Cependant, il faut remarquer que les statistiques peuvent avoir de limitations si pendant la collecte des données sur ce phénomène, elles sont cachées ou bien, comme mesure de protection des adolescentes au moment de témoigner, elles sont omises.

Mots clés: Relations illicites, grossesse chez adolescents, abus de pouvoir, statistiques de population adolescente, loi des relations inconvenantes.

\section{Referencias bibliográficas}

Asamblea Legislativa de la República de Costa Rica. Proyecto de Ley 19.337, del 30 de setiembre, sobre reforma de los artículos 159 y 161 de la Ley n. ${ }^{\circ} 4573$, Código Penal; reforma de los artículos 14,16 y 158 y derogatoria de los artículos 21, 22, 36 y 38 de la Ley n. ${ }^{\circ}$ 5476, Código de Familia; reforma del artículo 39 de la Ley n. ${ }^{\circ} 63$, Código Civil y reforma del artículo 89 de la ley n. ${ }^{\circ} 3504$, Ley orgánica del Tribunal Supremo de Elecciones y del Registro Civil, para el fortalecimiento de la protección legal de niñas y adolescentes mujeres, ante situaciones de violencia de género asociadas a relaciones abusivas. Asamblea Legislativa expediente n. ${ }^{\circ}$ 19.337, 2014. Acceso: 26 de marzo, 2020. http://www.asamblea.go.cr/sd/SiteAssets/Lists/ Consultas $\% 20$ Biblioteca/EditForm/Proyecto-19337\%20relaciones $\% 20$ menores $\% 20 \mathrm{de} \% 20$ edad.pdf

Asamblea Legislativa de la República de Costa Rica. Ley 9406, 13 de enero, para el fortalecimiento de la protección legal de las niñas y las adolescentes mujeres ante situaciones de violencia de género asociadas a relaciones abusivas, La Gaceta, alcance número 9 del 2017. Acceso: 26 de marzo, 2020. http://www. pgrweb.go.cr/scij/Busqueda/Normativa/Normas/nrm_texto_completo.aspx?param1=NRTC\&nValor1=1 \&nValor2=83353\&nValor3=106995\&strTipM=TC

Asamblea Legislativa de Costa Rica. Código de Familia de Costa Rica, 1973. Acceso: 26 de marzo, 2020. http://www.pgrweb.go.cr/scij/Busqueda/Normativa/Normas/nrm_texto_completo. aspx?nValor $1=1 \&$ nValor $2=970$

Fernández Carballo, Rodolfo y Andrea Duarte Cordero. «Preceptos de la ideología patriarcal asignados al género femenino y masculino, y su refractación en ocho cuentos utilizados en el Tercer Ciclo de la Educación General Básica del Sistema Educativo Costarricense en el año 2005». Revista Educación 30 (2) (2006). Acceso: 26 de marzo, 2020. https://revistas.ucr.ac.cr/index.php/educacion/article/view/2235

Fondo de Población de Naciones Unidas. Uniones Tempranas y Embarazo en la Niñez y la Adolescencia en Costa Rica (2014). Acceso: 26 de marzo, 2020. http://tiempodehablar.paniamordigital.org/files/Descargas/ files/4_221_unionesimpropiasunfpapaniamor2014.pdf

Instituto Nacional de Estadística y Censos (INEC) de Costa Rica. Estadísticas vitales 2000-2018. Acceso: 26 de marzo, 2020. http://inec.cr/sistema-de-consultas

León Camacho, Jennyfer Akira. «Propuesta educativa en materia de violencia contra las mujeres en relaciones de pareja, dirigida a centros educativos, comunidades y oficiales de la Fuerza Pública: un abordaje por medio de la oficina de la mujer del cantón central de Limón» (tesis de licenciatura en Psicología, Universidad de Costa Rica, 2018): 4. Acceso: 26 de marzo, 2020. http://repositorio.sibdi.ucr.ac.cr:8080/xmlui/ handle/123456789/6550 
Montesó Curto, Pilar. «Dificultades para el avance de las mujeres. Diferentes teorías sociológicas». Enfermería Global, Vol. 13, Núm. 4 (2014): 266. Acceso: 26 de marzo, 2020. http://revistas.um.es/eglobal/article/ view/186761

Observatorio de violencia de género contra las mujeres y acceso a la justicia, Poder Judicial. Estadísticas casos entrados netos en el Ministerio Público (penal adultos). Poder Judicial de Costa Rica. 2019. Acceso: 26 de marzo, 2020. https://observatoriodegenero.poder-judicial.go.cr/soy-especialista-y-busco/estadisticas/ delitos-sexuales/

Villarreal Montoya, Ana Lucía. «Relaciones de poder en la sociedad patriarcal». Revista Electrónica Actualidades Investigativas en Educación, vol. 1, no. 1 (2001): 3. Acceso: 26 de marzo, 2020. https://revistas.ucr. ac.cr/index.php/aie/article/view/8456 